\title{
El español en la red
}

Mabel Giammatteo, Patricia Gubitosi, Alejandro Parini (eds.) (2017).

Madrid/Frankfurt: Iberoamericana/Vervuert, 334 páginas.

ISBN 978-84-16922-45-1 (Iberoamericana). ISBN 978-3-95487-737-9 (Vervuert).

ISBN 978-3-95487-738-6 (e-Book).

\section{( Julio César Sal Paz}

Universidad Nacional de Tucumán - Consejo Nacional de Investigaciones Científicas y Técnicas, Argentina jsalpaz@gmail.com

El estudio de las transformaciones que las tecnologías de la información y la comunicación (TICs) ejercen sobre las prácticas discursivas y sus condiciones de producción y recepción ha despertado, a lo largo del tiempo, la atención y curiosidad de la comunidad científica, en general y de las ciencias sociales, en particular. Prueba de ello es el florecimiento de diferentes interdisciplinas -como la cibercultura, las humanidades digitales, la ciberpragmática, el análisis del discurso mediado por ordenador o la retórica digital- que se han insertado con ímpetu en su interior para propiciar, a través de reflexiones teóricas y aparatos metodológicos y críticos variados, un mayor entendimiento de la complejidad inherente a los procesos comunicativos de la Sociedad de la Información y el Conocimiento (SIC).

Ahora bien, a pesar de que en el Informe 2018, El español: una lengua viva, elaborado por el Instituto Cervantes, se explica que el idioma en el que fue escrito el Quijote se ubica, actualmente y a nivel planetario, como la tercera lengua más utilizada en Internet y como la segunda más usada en redes sociales, en el ámbito de la lingüística hispánica, se han publicado hasta la fecha pocas investigaciones panorámicas que den cuenta de este fenómeno, de sus particularidades y complejidad. En este sentido, la aparición de El español en la red, editado por Mabel Giammatteo (Universidad de Buenos Aires), Patricia Gubitosi (Universidad de Massachusetts Amherst) y Alejandro Parini (Universidad de Belgrano y Universidad de Buenos Aires), representa, precisamente, una intervención lúcida y activa sobre esta área de vacancia. El libro reúne trece contribuciones de diecisiete destacados especialistas, procedentes de diferentes latitudes, que abordan, a través de múltiples miradas, perspectivas teóricas y marcos conceptuales diversos, la situación actual de los intercambios comunicativos digitales en lengua española, analizados desde un proceso de reflexión sistemático, riguroso, preciso, multifacético y prolífero.

La obra-vol. 68 de la colección "Lingüística Iberoamericana”- se inicia con una introducción, integrada por un prólogo de los editores, presentado bajo el rótulo de "La comunicación mediada por computadora" y por un trabajo de Crispin Thurlow, catedrático de la Universidad de Berna, titulado "Enmarcando el lenguaje de los nuevos medios". Ambos estudios operan como anclaje contextual para el conjunto de investigaciones que se estructuran alrededor de tres secciones, puesto que articulan la descripción de ciertas propiedades comunes, inmanentes al soporte y a los dispositivos, que permiten postular la existencia de unos parámetros interaccionales estables que sirven para puntualizar la categoría de discurso digital, hilo conductor de toda la propuesta.

La primera parte, denominada "Géneros y estilos en la red", problematiza acerca de una de las consecuencias de la aplicación de las tecnologías digitales a las distintas esferas de la praxis social: el surgimiento de nuevos géneros discursivos, de inéditos modos de interacción social. Son, en efecto, auténticos sistemas de organización del comportamiento comunicativo, puesto que evidencian las formas en que los grupos y las comunidades definen y resignifican las actividades sociales a través del discurso, pautando los procedimientos de autofiguración y de representación del otro y de la realidad.

En primer término, Ana Pano Alamán (Universidad de Bolonia), en "La intensificación en los blogs de contenido cultural de la prensa digital española y argentina", formaliza un estudio cualitativo, exploratorio y contrastivo de seis bitácoras culturales sobre cine y literatura, alojadas en las versiones online de los periódicos El País y La Nación. Para ello, centra su análisis tanto en las entradas o post como en los comentarios que estos generan como respuesta. La información y la opinión prevalecen en el género; son las intenciones discursivas que se canalizan mediante mecanismos de intensificación, construidos a partir de rasgos coloquializadores y conversacionales que aportan afectividad, conectividad e interactividad. A partir de la determinación de los condicionantes del medio y de la situación comunicativa propia 
de los weblogs de diarios hegemónicos, aborda la intensificación como una categoría pragmática y su incidencia en los planos del enunciado, de la enunciación y del proceso de interacción entre autores de post y usuarios. Las conclusiones a las que arriba revelan una marcada presencia de intensificadores en los comentarios respecto a las entradas, donde la ocurrencia es de menor alcance. Esta orientación se acentúa para los ejemplos argentinos. En el interior de estas intervenciones operan dispositivos evaluativos que provocan proximidad, inmediatez y brevedad para vehiculizar opiniones en un diálogo adyacente al polo del registro coloquial.

A continuación, en "La realidad sintáctica de Twitter. Subordinación en 140 caracteres", Álvaro Recio Diego y Carmela Tomé Cornejo, investigadores de la Universidad de Salamanca, focalizan el interés en la sintaxis del español digital utilizado en el género tuit, en un corpus conformado por 300 casos seleccionados a partir de los trending topics con mayor impacto en España durante el primer semestre del año 2014. Los resultados exhiben un evidente predominio de la composición oracional frente a la oración simple o los enunciados nominales, que los especialistas interpretan como reflejo de la lengua general, en su modalidad oral o escrita. Es decir, esta estructuración sintáctica posibilitaría una mayor eficacia comunicativa. La subordinación -sustantiva y de relativo, por su rol argumental y restrictivo, respectivamente- se impone a la coordinación y a la yuxtaposición, que aparece con más asiduidad, hecho que aproximaría estos intercambios al modo oral, ligado con la concepción del género como una gran conversación online, en marcha o en construcción.

Finalmente, esta sección se cierra con la contribución de Lucía Cantamutto (CONICET - Universidad Nacional del Sur y Universidad Nacional del Comahue), titulada "Economía, claridad y expresividad lingüísticas: el estilo comunicativo digital del teléfono móvil en el español bonaerense". La autora parte de la definición genérica de los SMS, para luego dar cuenta del estilo comunicativo en los dominios de familia, de pareja, de relación social, del ámbito educativo y de la esfera laboral, en una muestra recolectada en Bahía Blanca, integrada por 6.200 mensajes de texto de la variedad dialectal del español bonaerense. En su análisis contempla las dimensiones pragmático-discursiva, léxicosemántica y morfosintáctica. Los hallazgos obtenidos demuestran que el estilo comunicativo digital en los SMS -caracterizado por las variables economía, expresividad y claridad-, para generar sentidos, apela a elecciones simultáneas y consecutivas que intervienen, tanto en un nivel macro, a través de la selección de una plataforma, como en el micro, por medio de las opciones lingüísticas que escogen los interlocutores durante la interacción. Es decir, la configuración triádica debe entenderse como realizaciones dinamizadoras que tienden a uno u otro polo. Así, por ejemplo, el dominio de la amistad, se acerca a la expresividad; el familiar, a la claridad, mientras que todos los examinados permanecen vinculados de manera estable a la brevedad, rasgo constitutivo del género y de los dispositivos utilizados para su concreción.

“Multilingüismo en la red", nombre de la segunda parte del libro que reseñamos, agrupa cuatro trabajos que indagan acerca del cambio de código, el bilingüismo en situación de diglosia y la interacción digital en diversos ámbitos de uso y esferas de prácticas discursivas. Las lenguas actúan como un poderoso motor de difusión de culturas y tradiciones, e internet como uno de los principales vehículos de intercambio de información y conocimiento. Sin embargo, según un reciente documento publicado por la UNESCO (2015), existen muchos idiomas que no tienen presencia en la red, razón por la cual artículos como los que componen esta sección representan la posibilidad de concienciar sobre la importancia que reviste el empleo de lenguas maternas minoritarias en los nuevos dispositivos para, de este modo, contribuir a su preservación.

Alba Arias Álvarez de la Universidad de Massachusetts Amherst, en "Cambio de código en la red: la expresión de la identidad en Asturias", analiza este fenómeno, desde las lógicas cuantitativa y cualitativa, en 970 comentarios extraídos, entre mayo y junio de 2015, de cuatro páginas públicas de Facebook, relacionadas con la cultura y la actualidad de esta comunidad bilingüe. Los resultados alcanzados indican que el cambio de código del español al asturiano en esta red social asume un rol estratégico que posibilita a los administradores y comentaristas visibilizar en el discurso digital la identidad asturiana, a través de un uso cargado de expresividad, para instruir sobre tópicos vinculados con la tradición de la región.

Por su parte, en "Red: diccionarios, traductores y foros", Montserrat Casanovas Catalá y Yolanda Capdevilla-Tomàs (Universidad de Lleida) exploran, amparadas en los postulados de los nuevos estudios sobre literacidad, cómo alumnos universitarios incluyen en el proceso de escritura académica, en catalán, español e inglés, algunas herramientas digitales de la web 2.o. A partir del diseño de un cuestionario, con preguntas mayoritariamente cerradas, administrado como prueba piloto a una muestra constituida por 58 estudiantes de cuarto año de la carrera de grado de 
Educación Primaria de la Universidad de Lleida, las especialistas concluyen que la población observada, por una parte, emplea recursos electrónicos (diccionarios y traductores), fundamentalmente, en desmedro de obras en papel y, por la otra, que acude a sitios multifuncionales para efectuar consultas lexicográficas y lingüísticas diversas -casi exclusivamente a Google, sistema de gran versatilidad-, al momento de encarar la redacción de géneros disciplinares del ámbito universitario.

Patricia Gubitosi (Universidad de Massachusetts Amherst), una de las coordinadoras del volumen, en "Cambio de código y mensajes de texto: diálogo bilingüe intergeneracional”, inspecciona, desde el análisis conversacional, un corpus de 737 SMS, enunciados por cuatro hablantes procedentes de Argentina, de sexo femenino -madres e hijas adolescentes-, radicadas en Estados Unidos, a fin de observar la estrategia discursiva de cambio de código entre el español y el inglés, presente en las intervenciones de las participantes. Las derivaciones de la investigación indican que el fenómeno en el contexto digital está condicionado por diversas variables, motivo por el cual debe ser apreciado desde las particularidades de los hablantes involucrados en las situaciones comunicativas concretas, tal como ocurre en las interacciones no mediadas.

Por último, clausura el segundo eje del libro, el ensayo "Contextos y sentidos de las prácticas escritas bilingües entre jóvenes wichis" de Camilo Ballena, docente de la Universidad de Chaco Austral, y Virginia Unamuno, investigadora de CONICET y profesora de la Universidad Nacional de San Martín. En la comunicación, los autores se proponen indagar en Facebook la combinación y/o alternancia entre el wichi y el español de la Argentina en la escritura de jóvenes y adultos bilingües de Chaco, a partir del estudio sociolingüístico y conversacional de un corpus de datos multimodal, conformado por 68 entradas y 123 comentarios, efectuados en la red social entre julio de 2014 y junio de 2015. Las derivaciones del trabajo demuestran que los espacios digitales de interacción, privados y (semi) públicos, configurados en la plataforma, promueven el bilingüismo con diferentes propósitos pragmáticos vinculados con los potenciales destinatarios de la comunicación y fomentan el empleo escrito de la lengua minoritaria como manifestación de la identidad etnolingüística de sus hablantes.

Finalmente, el último bloque, titulado "Contexto, participación e interacción en la red”, reúne cuatro capítulos que giran en torno a los comportamientos comunicativos de los usuarios en diferentes espacios y entornos digitales. El primero de ellos, "Adecuación del discurso al contexto institucional en un foro virtual universitario", corresponde a Eliana Lucián Vargha, de la Universidad de la República. En él, se efectúa un examen cualitativo de las interacciones verbales producidas en el seno de una comunidad de práctica con 63 participantes -62 estudiantes y un docente-, quienes participan, durante abril de 2010 y mayo de 2011, de un foro de discusión que opera como apoyatura del curso presencial “Taller l”, asignatura de la Tecnicatura Corrección de estilo de la Udelar. Los resultados del artículo determinan que este género académico, gestado en los nuevos medios, actúa como un microcosmo con normas propias de funcionamiento. En este contexto institucional, se acuerdan posiciones y roles, a través del empleo de estrategias discursivas particulares que requieren el desarrollo de competencias lingüísticas, pragmáticas y tecnológicas para garantizar el logro comunicativo.

A continuación, Cristina Vela Delfa, profesora de la Universidad de Valladolid, en el trabajo "Coherencia, cohesión y estructura de la interacción en el discurso digital: un análisis de los intercambios en la red social Facebook" aborda estos fenómenos lingüísticos, a partir de la observación participante del muro de dos usuarios, con sus post y comentarios, efectuada a lo largo de diez días no consecutivos de actividad dentro del marco de cinco meses del año 2015. La investigación proyecta como conclusiones que los mecanismos discursivos de coherencia y cohesión son disímiles al de los de los textos del universo papel, pues están condicionados y beneficiados por las características constitutivas del soporte, tales como la influencia multipantalla, la hiperconectividad, la multimodalidad y la hipertextualidad.

Por su parte, en "Emoticonos y cortesía en los mensajes de WhatsApp en España", Agnese Sampietro (Universidad de Valencia) reflexiona sobre el uso de los emoticonos y los emojis en la comunicación en la aplicación de WhatsApp en España. Para ello, examina un corpus textual conformado por 50 conversaciones online en 49 diadas de usuarios diferentes, a partir de una taxonomía de categoría de cortesía proveniente de la sociopragmática. Los resultados ofrecidos señalan que los emoticonos se emplean en la popular aplicación como recursos para coadyuvar con el juego entre autonomía y filiación, propio de la cortesía estratégica, presente en las interacciones interpersonales.

Para cerrar este apartado, y toda la obra, Manuel Alcántara-Plá, catedrático de la Universidad 
Autónoma de Madrid, aborda en "El contexto de los mensajes en la comunicación digital”, un estudio de caso relacionado con un escándalo político originado por la publicación de un mensaje con contenido inapropiado en la red de microblogging Twitter, por parte de un funcionario público. Las conclusiones brindadas se vinculan con el poder de las palabras para configurar procesos de contextualización, descontextualización y recontextualización en los diferentes dispositivos digitales.

Anhelamos que este libro contribuya con la discusión del rol del español en la red, que se constituya en aporte a su entendimiento y sea recibido por los lectores hispanohablantes y por la comunidad científica en general con calidez y beneplácito, pues se trata de una propuesta introductoria, actualizada y rigurosa, dirigida a una amplia variedad de lectores con diferente formación. Entrega luces sobre diversos géneros del ciberespacio, abordados con categorías de análisis heterogéneas y multidisciplinarias. En suma, es una obra esencial que auguramos se convertirá, sin duda, en un punto de partida para cualquier investigación que se relacione, en general, con las características que adopta la lengua española mediada por las nuevas tecnologías, empleada en sus variadas plataformas y pantallas.

\section{Bibliografía}

"Instituto Cervantes (2018). El español: una lengua viva. Informe 2018. Madrid: Instituto Cervantes.

» UNESCO (2015). Una década de promoción del plurilingüismo en el ciberespacio. París: UNESCO. 